Invited Paper

\title{
Biological applications of an LCoS-BASED PROGRAMMABLE ARRAY MICROSCOPE (PAM)
}

\author{
Guy M. Hagen ${ }^{\mathrm{a}}$, Wouter Caarls ${ }^{\mathrm{b}}$, Martin Thomas ${ }^{\mathrm{c}}$, Andrew Hill ${ }^{\mathrm{c}}$, \\ Keith A. Lidke ${ }^{\mathrm{d}}$, Bernd Rieger ${ }^{\mathrm{b}}$, Cornelia Fritsch ${ }^{\mathrm{e}}$, Bert van Geest ${ }^{\mathrm{f}}$, \\ Thomas M Jovin $^{\mathrm{a}}$ and Donna J. Arndt-Jovin ${ }^{\mathrm{a}^{*}}$ \\ a Department of Molecular Biology, Max Planck Institute for Biophysical Chemistry, \\ Am Faßberg 11, 37077 Göttingen, Germany; ${ }^{b}$ present address: Quantitative Imaging Group, \\ Department of Imaging Science \& Technology, Delft University of Technology, Delft, The \\ Netherlands; ' Cairn Research, Ltd., Graveney Road, Faversham, Kent, UK; \\ d present address: University of New Mexico, Dept. of Physics, Albuquerque 87131, New Mexico; \\ epresent address: School of Life Sciences, University of Sussex, Brighton, UK; \\ ${ }^{f}$ Lambert Instruments, Turfweg 4, 9313 TH Leutingewolde, The Netherlands
}

\begin{abstract}
We report on a new generation, commercial prototype of a programmable array optical sectioning fluorescence microscope (PAM) for rapid, light efficient 3D imaging of living specimens. The stand-alone module, including light source(s) and detector(s), features an innovative optical design and a ferroelectric liquid-crystal-on-silicon (LCoS) spatial light modulator (SLM) instead of the DMD used in the original PAM design. The LCoS PAM (developed in collaboration with Cairn Research, Ltd.) can be attached to a port of a(ny) unmodified fluorescence microscope. The prototype system currently operated at the Max Planck Institute incorporates a 6-position high-intensity LED illuminator, modulated laser and lamp light sources, and an Andor iXon emCCD camera. The module is mounted on an Olympus IX71 inverted microscope with 60-150X objectives with a Prior Scientific $x, y$, and $z$ high resolution scanning stages. Further enhancements recently include: (i) point- and line-wise spectral resolution and (ii) lifetime imaging (FLIM) in the frequency domain. Multiphoton operation and other nonlinear techniques should be feasible.

The capabilities of the PAM are illustrated by several examples demonstrating single molecule as well as lifetime imaging in live cells, and the unique capability to perform photoconversion with arbitrary patterns and high spatial resolution. Using quantum dot coupled ligands we show real-time binding and subsequent trafficking of individual ligand-growth factor receptor complexes on and in live cells with a temporal resolution and sensitivity exceeding those of conventional CLSM systems. The combined use of a blue laser and parallel LED or visible laser sources permits photoactivation and rapid kinetic analysis of cellular processes probed by photoswitchable visible fluorescent proteins such as DRONPA.
\end{abstract}

Keywords: fluorescence microscopy, confocal, emCCD, SLM, quantum dots, EGFR, erbB, DRONPA, FLIM, FRET

* corresponding author: djovin@,gwdg.de, tel. +49-551-2011393, fax +49-551-2011467

Imaging, Manipulation, and Analysis of Biomolecules, Cells, and Tissues V, edited by Daniel L. Farkas, Robert C. Leif, Dan V. Nicolau, Proc. of SPIE

Vol. 6441, 64410S, (2007) - 1605-7422/07/\$18 - doi: 10.1117/12.710995

Proc. of SPIE Vol. 644164410 S-1 


\section{INTRODUCTION}

\subsection{The biological system of erbB receptor tyrosine kinases}

The erbB family of receptor tyrosine kinases (RTKs) includes erbB1 (the classical epidermal growth factor (EGF) receptor, hereafter referred to as EGFR), erbB2, erbB3 and erbB4. Activation of these transmembrane proteins controls numerous cellular processes such as DNA replication, motility and differentiation. Binding specific peptide ligands to the ectodomains of the RTKs leads to auto- and transactivation of the cytoplasmic protein kinase domains. The activated receptors are recognized by adaptor proteins, inducing several signal transduction cascades such as those mediated by MAP kinases. The fate of the activated receptors is complex: endocytosis via coated pits, covalent modification. (deactivation by enzymatic dephosphorylation and ubiquitinylation), and endosomal trafficking leading either to proteosomal and/or lysosomal degradation or to recycling to the plasma membrane. The overexpression and unrestrained activation of the erbB family are implicated in many types of cancer [1].

\subsection{Semiconductor-core quantum dots for single molecule studies on live cells}

We have shown in previous publications that quantum dots (QDs) bearing natural ligands function as effector molecules and provide the means for prolonged real-time visualization of erbB molecules on living cells. One can follow the multiple steps of the signaling pathways and generate detailed movies of the underlying molecular processes (available as supplementary information in references [2-4].)

For the studies reported here, biotinylated EGF was bound to commercial streptavidin conjugated QDs. QDs have unique features providing many advantages for cellular imaging: (i) high absorption cross-sections and quantum yields, permitting detection down to the single nanoparticle level and reliable quantitation of binding and transport phenomena; (ii) extreme photostability, allowing imaging over prolonged periods; (iii) broad excitation spectra rising toward the UV, allowing the simultaneous excitation of visible fluorescent proteins (VFP) and QDs; and (iv) narrow emission bands across the visible spectrum. QDs can be regarded as "single molecule" probes.

\subsection{The Programmable Array Microscope (PAM)}

The programmable array microscope (PAM) conceived and developed in the Department of Molecular Biology of the Max Planck Institute for Biophysical Chemistry [5-10] is a powerful tool combining the capabilities of previously described optical sectioning techniques in a single microscope. The defining feature of a PAM is the presence of a spatial light modulator (SLM) in the image plane, where it is used to define a pattern of conjugate illumination and detection. The family of PAMs include optical sectioning systems operating in fluorescence and reflection modes, as well as spectroscopic imaging systems [5-8, 11-13]. In addition, the programmable SLM allows the implementation of flexible photoactivation and photodepletion strategies [14].

The conjugate image (Ic) is formed by light originating from the object plane and returning along the optical path of the illumination. The non-conjugate image (Inc) comprises fluorescent light impinging on pixels of the SLM not used for sample illumination, and thus represents primarily out-of-focus contributions. A PAM provides much more temporally efficient excitation than CLSMs, attaining up to 50\% coverage of the SLM (and the sample) compared with single point raster systems. The detection is equally superior, by virtue of collection of all the emission and use of close to ideal emCCD detectors with negligible readout noise (due to the electron multiplication technology). The PAM can be configured dynamically for a wide range of data acquisition and analysis strategies. Rapid diffraction-limited optical sectioning is achieved with pseudorandom (Sylvester) sequence scans and real-time joint processing of the conjugate and non-conjugate images [11-13, 15]. Hardamard transform techniques permit 3D spectroscopic imaging [12, 16, 17] and frequency-domain FLIM is also feasible $[17,18]$ (this report). An operational comparison of the PAM and CLSMs is given in Table 1. 
Table 1. Performance of the PAM compared with a raster scan CLSM in terms of relative parameters.

\begin{tabular}{|lll|}
\hline \multicolumn{1}{|c|}{ Parameter } & PAM/CLSM & \\
$a$ relative irradiance at the focal plane & $10^{-2}$ & full-field vs. focused laser \\
$b$ relative detector quantum efficiency & 10 & CCD vs PMT \\
$c$ relative dwell time per pixel & $10^{3}$ & PAM, 0.3-10 ms; CLSM $10 \mu \mathrm{s}$ \\
$d$ relative number of scanning apertures & $10^{4}$ & $\sim 10^{5}$ pixels \\
relative signal strength (conjugate $=$ infocus) & & \\
$\quad 10^{2}$ & \\
$\quad$ grid $(a \bullet b \bullet c)$ & $10^{3}$ & dual signal vs. pinhole \\
pseudorandom sequence $(a \bullet b \bullet c \bullet n / 2)$ & $10^{2}$ & \\
Proportional increase in speed $(d / c)$ & 10 & \\
\hline
\end{tabular}

\section{MATERIALS AND METHODS}

\subsection{Reagents}

Biotin-EGF, EGF, and streptavidin conjugated, pegylated QDs were from Invitrogen. PD153035 was purchased from Calbiochem. Live cell labeling was carried out in Tyrode's buffer with $20 \mathrm{mM}$ glucose and $0.1 \%$ BSA. PBS is phosphate buffered saline.

\subsection{Cell lines}

A431 cells, an epidermal carcinoma cell line expressing $2 \times 10^{6}$ EGFRs per cell, the same cell line stably transfected with EGFR-eGFP, CHO stably transfected cells expressing $6 \times 10^{5}$ EGFR-eGFP receptors, and trasnfected A431 cells stably expressing $3 \times 10^{5}$ erbB3-DRONPA were maintained in DMEM with $10 \%$ fetal calf serum.

\subsection{Cell treatments}

Cells were typically starved $(0.1 \%$ FCS $)$ overnight. When appropriate, cells were treated with $1 \mu \mathrm{M}$ PD153035 EGFR kinase inhibitor for $2 \mathrm{hr}$ at $37^{\circ} \mathrm{C}$ prior to and during diffusion measurements.

\subsection{QD conjugation to epidermal growth factor}

Monobiotinylated-EGF (b-EGF) was coupled to the streptavidin QDs by incubation with $10 \mathrm{nM}$ QDs in PBS containing $1 \%$ BSA for at least 30 min at $4{ }^{\circ} \mathrm{C}$. at $3: 1$ or 1:1 molar ratios unless otherwise specified.

\subsection{PAM}

The new generation programmable array microscope (PAM) is a commercial prototype developed in collaboration with Cairn Research Ltd. (Faversham, Kent, UK). The stand-alone module, including light source(s) and detector(s), features a compact optical configuration incorporating a ferroelectric liquid-crystal-on-silicon (LCoS, SXGA-R2D, Forth Dimension Displays, Dunfermline, Scotland) SLM instead of the DMD used in the first PAM designs of our laboratory. The LCoS-based PAM can be attached to a camera port of any unmodified fluorescence microscope. The prototype system currently operated at the Max Planck Institute for Biophysical Chemistry (Göttingen, Germany) incorporates a 6-position high-intensity LED illuminator as well as digitally modulated laser light sources (both diode lasers and AOM-modulated argon-ion lasers), and an Andor iXon emCCD camera. A lamp-based system incorporating ferroelectric shutters is also in operation. The system is mounted on an Olympus IX71 inverted microscope with 60$150 \times$ objectives, a high precision $x, y, z$ stage (Prior, Nanoscan Z), and high speed filter wheels (Prior Scientific, Cambridge, UK) . 


\subsection{Software and image processing}

\subsubsection{Real time optically-sectioned imaging with the PAM}

In the LCoS-based PAM, the conjugate and the non-conjugate images are recorded simultaneously side-by-side with a single CCD camera. To yield a properly optically sectioned image, subtraction of these images is required. Before acquisition, the two images are registered by a search procedure using a fixed step size optimization of the 2dimensional correlation coefficient and the registration parameters are translation (step size of 0.1 pixels), rotation (step size 0.001 radians), and magnification (step size 0.001). During acquisition, a background image is first subtracted from the image pair. Then, the non-conjugate image is transformed to overlap the conjugate image by applying the established registration parameters. For subpixel transformations, bilinear interpolation is used. The images are then subtracted using a weighting factor which is dependent on the duty cycle of the pattern used for acquisition [13]. The final image is scaled, and offset removed. If desired, a Gaussian filter with an adjustable standard deviation is applied. In order to perform the transformation and subtraction with sufficient speed (i.e. for real-time display and interactive parameter adjustment), the operations are carried out on a NVidia Quadro FX 4400 GPU board using the BrookGPU GPU library and DirectX 9 runtime. The GPU board is coupled to an Intel Xeon $3.2 \mathrm{GHz}$ processor. By offloading the image processing to the GPU, the computation time is less than the fastest possible PAM exposure time required for a full scan ( $\sim 16 \mathrm{~ms}$ for the SXR2 LCoS). Optical sectioning by the PAM is thus real-time, i.e. the conjugate, nonconjugate and processed, sectioned images are displayed at video rate on the screen.

\subsubsection{Single particle tracking in the PAM}

Data for single particle tracking (SPT) were acquired using the PAM displaying pseudo-random sequence patterns for the acquisition of conjugate and non-conjugate images at up to $20 \mathrm{~Hz}$ (400 frames, $16 \mathrm{~ms}$ exposure). In some cases QDEGF labeling was performed in the presence of a kinase inhibitor, PD153035 that inhibits the activation and dimerization of the EGFR receptor. In other cases, a very short labeling (1 to 2 min) of the cells with 100 pM QD-EGF followed by washing allowed binding to isolated (monomeric) EGFR which was subsequently tracked. We devised an automated method for selecting QDs for tracking and analysis that fit high intensity regions to a Gaussian profile approximating the point spread function (PSF) of the microscope. The quality of the fit was used to select a spot for analysis, allowing batch processing of many acquired series. The tracking routines were written in DIPimage (TU Delft, www.qi.tnw.tudelft.nl/DIPimage) a toolbox for Matlab (The Mathworks, Massachusetts, USA). The tracking with the processed conjugate images was performed offline, i.e. after acquisition.

\section{RESULTS}

In this paper we feature (i) the rapid, light efficient 3D imaging of the early binding events of individual QD-EGF ligands with a commercial prototype of a new generation, optically sectioning programmable array microscope (PAM); (ii) the diffusional behavior of non-activated single receptors on the cell body and filopodia using a high speed and high sensitivity electron multiplying CCD (emCCD) camera and conjugate imaging in the PAM; (iii) FLIM imaging with the PAM demonstrating FRET between activated EGFR receptors in confocal sections; and (iv) dual laser implementation in the PAM for photoconversion and monitoring of the erbB3 receptor coupled to DRONPA in live CHO cells.

\subsection{Programmable Array Microscopy}

Dual pass programmable array microscopes (PAMs) are defined by the use of a spatial light modulator (SLM) in a primary image plane of a standard fluorescence microscope. The SLM provides for structured illumination as well as conjugate descanning of the image to achieve optical sectioning. The major advantages of the PAM are: (i) simple, inexpensive design with no moving parts; (ii) speedup in optical sectioning due to an illumination duty cycle for each pixel of up to 50\%; (iii) optimal detection sensitivity, using emCCD cameras; (iv) continuously programmable, arbitrary, and adaptive optical sectioning modes between or within images using libraries of dot, line, or pseudo-random (Sylvester) sequence patterns; (v) efficient and sensitive optical sectioning due to simultaneous detection and processing of both conjugate and non-conjugate light; (vi) compatibility with polarization, hyperspectral, lifetime-resolved, and other imaging modes; and (vii) minimal photobleaching. 
Our first generation implementation of the PAM used a digital micromirror device (DMD) for optical sectioning or transmissive liquid crystal SLMs for imaging spectroscopy [5, 11-14, 16-19]. Other reported PAMs are based on DMD [20-22] and liquid crystal-on-silicon (LCoS) [23] SLMs. Of these other implementations, only one [21] was applied in fluorescence microscopy, using a fringe projection technique similar to that of Neil et al. [24]. Our DMD-based PAM suffered from several serious limitations leading to degradation of the acquired confocal images: lack of a suitable, easy to use video interface for scanning pattern definition, pronounced diffraction losses, limited VGA format, and a small micromirror tilt angle. In addition, the imaging of conjugate (in-focus light from "on" pixels) and non-conjugate (outof-focus light from "off" pixels) on a single camera was not feasible.

We have developed a new PAM in collaboration with Cairn Research Ltd. (Faversham, UK) based on a ferroelectric LCoS microdisplay (Forth Dimension Displays, Dunfermline, Scotland). This microdisplay has several favorable characteristics for use in a PAM: SXGA resolution, high fill factor (93\%), good contrast ( $>400: 1)$, and a fast liquid crystal switch time $(40 \mu \mathrm{s})$. When used for video projection, the microdisplay operates in a 24-bit colorsequential mode; each video frame is broken into 24 adjustable-length bitplanes, 8 each for red, green and blue color channels. For PAM operation, the color-sequential mode is disabled and each of the 24 bitplanes is displayed for the same length of time, refreshing at $1.44 \mathrm{kHz}$. Scanning patterns are defined by simple display of a 24-color Windows bitmap file via a standard computer DVI video interface. Possible scanning patterns include those based on dot lattices, line arrays, or pseudorandom (Sylvester) sequences or arrays $[13,19]$. An "on" pixel is one in which linearly polarized light is reflected with a $90^{\circ}$ rotation in the plane of polarization. "Off" pixels reflect light with no change in polarization state. Light sources (LEDs, lasers, lamps) must thus be linearly polarized, either intrinsically, or by means of a polarizing beam splitter integrated in the PAM. The illumination patterns are relayed to the microscope and the elicited fluorescence redirected to the SLM. Emitted light of both linear polarization states is reflected selectively by the SLM and directed via a beam splitter (Cairn design) such as to generate the conjugate (in-focus) and non-conjugate (out-offocus) images on the common detector, in our case an emCCD (Ixon DV885 or DV887, Andor Technology, Belfast, Northern Ireland).

Spinning disk confocal microscopes also employ conjugate detection; the optically sectioned images have an offset due to cross talk between the many pinholes. The PAM has the advantage that a scaled subtraction can be performed using both the conjugate and non-conjugate images to generate a confocal image with no DC offset $[13,25]$. In addition, the sectioning capability is fully programmable and can achieve very high duty cycles. To generate the final image, the conjugate and non-conjugate images are registered and subtracted, with scaling factors that depend on the selected pattern. Image registration, scaling, background subtraction, filtering, and other image processing operations are performed in real time (processing and display require $10 \mathrm{~ms}$ per image) using a workstation-class graphics card and BrookGPU, a graphics processing unit (GPU) programming library freely available on the Internet [26]. Figure 1 shows a block diagram of the multipurpose microscope plus PAM module that was used in these experiments as well as a photograph of the inner construction of the PAM unit. 
A

Programmable Array Microscope (Module)

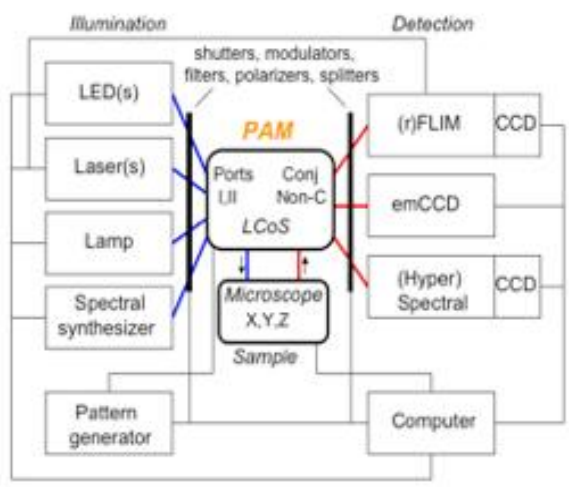

$\mathrm{B}$

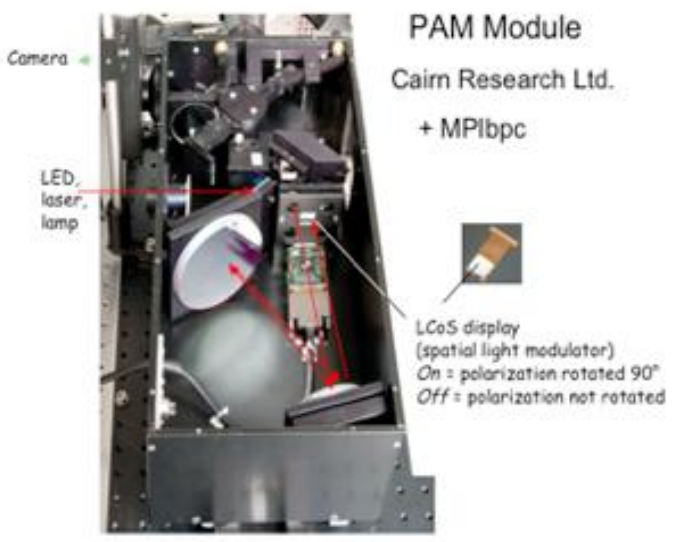

Figure 1. Features of the Programmable Array Microscope (PAM). (A) Modules comprising a system for high-speed, highly sensitive, multiparametric optical sectioning fluorescence microscopy of living cells. (B) Internal construction of PAM module. The heart is a Liquid Crystal on Silicon (LCoS) spatial light modulator for generating patterns of

The performance of the PAM for sectioning (60×/1.45 N.A. objective) was evaluated with a thin $(10 \mathrm{~nm})$ spincoated layer of rhodamine $6 \mathrm{G}$ in polyvinyl alcohol. The conjugate and non-conjugate intensity $z$-scan traces are plotted with the difference signal, corresponding to the true, diffraction-limited, sectioned output.
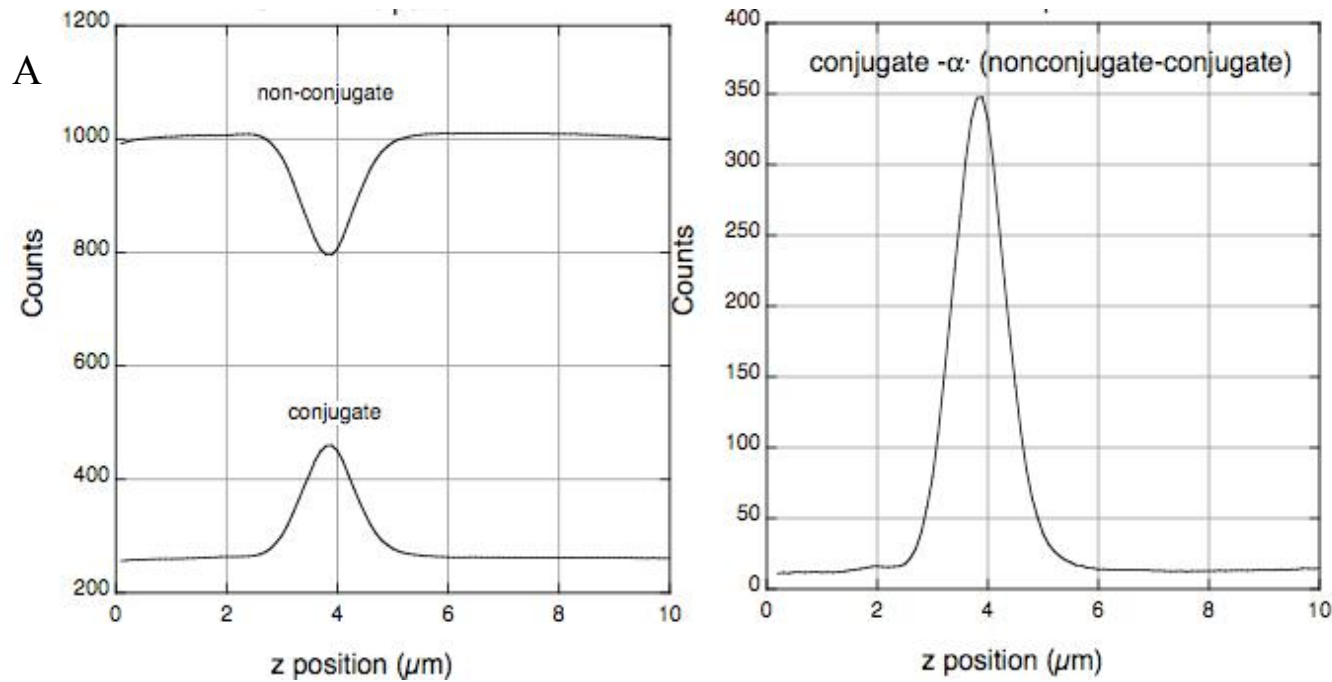

B

Figure 2. Traces of the intensities of the conjugate, non conjugate and scaled, subtracted PAM images of a $Z$ scan of 100 sections spaced by $0.1 \mu$ through a $10 \mathrm{~nm}$ fluorescence film of rhodamine 6G taken with the PAM. A. Conjugate and non conjugate traces. B. normalized difference image. FWHM $=1 \mu \mathrm{m}$. Excitation pattern: 8-24-1 (line thickness, period, and bitplane increment in pixels) line pattern. 


\subsection{Single QD imaging}

We made measurements of the initial steps of QD-EGF binding to EGFR on the filopodia and cell body and traffiking of QD-EGFR complexes in 3 dimensions. To demonstrate the sensitivity and high speed optical sectioning ability of the PAM, we imaged single QDs bound to A431 cells expressing the EGFR-eGFP. The QDs were added at a concentration of $100 \mathrm{pM}$ and allowed to bind for $3 \mathrm{~min}$ at room temperature. Excess QD-EGF was removed by washing with Tyrode's buffer. Imaging was started immediately at a rate of $\sim 6 \mathrm{~Hz}$ using an exposure time of $16 \mathrm{~ms}$. Three selected time frames from one optical section are shown in Figure 3.

Figure 3. Binding of QD-EGF and trafficking of QD-EGF-EGFR on an EG33-A431 cell imaged with the PAM. Three real-time processed images acquired with two colors from a series of 185 time points taken of 655 QD-EGF binding to an EG33-A431 cell (frames separated by $25 \mathrm{~s}$, starting at 2 minutes after binding was initiated). Excitation, $488 \mathrm{~nm}$; emissions, $520 \mathrm{~nm}$ HW30 and $655 \mathrm{~nm}$ HW20; objective, 150x/1.45NA. (upper panels, EGFR-eGFP; lower panels, 655-QD).
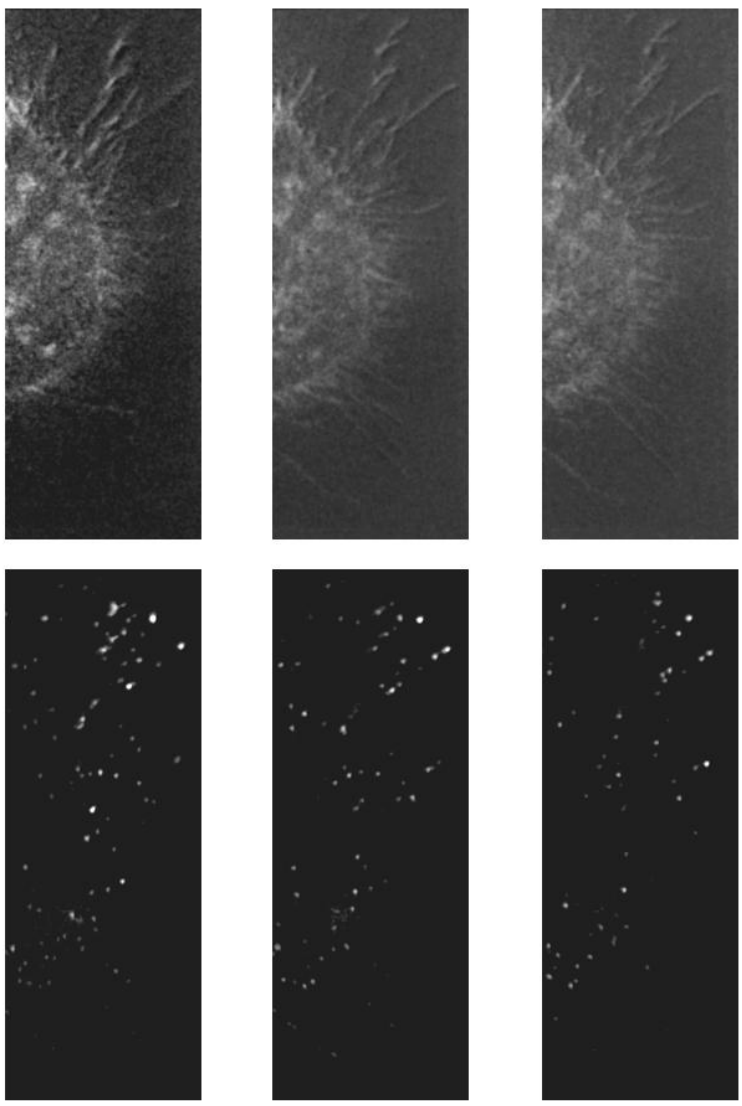

\subsection{Single QD-EGF-EGFR tracking}

Measurements of QD-EGF-EGFR diffusion using single particle tracking methods in laser scanning confocal microscopes yielded diffusion constants an order of magnitude smaller than those reported in studies of EGF-EGFR based on fluorescence recovery after photobleaching (FRAP) or fluorescence correlation spectroscopy (FCS) [3]. Here are presented new data acquired with the PAM and a fast emCCD camera of QD-EGF-EGFR under conditions permitting visualization of single particles.

Although QD blinking can be advantageous, i.e. for identifying single particles and achieving spatial superresolution [27], the phenomenon complicates the analysis of tracking due to the transient interruption of the signal. We have developed special data processing routines to take into account random QD blinking periods and to achieve automated identification and tracking of the QD-EGF-EGFR complexes ([15] and K.A.Lidke, et al., in preparation).

We originally used the algorithms on images acquired at video rates in a wide-field microscope of QD-EGF-EGFR complexes on the cell body and filopodia of cells treated with the kinase inhibitor PD153035, which inhibits transport and downstream signaling. Analysis of the trajectories from single QDs yielded mean square displacement (MSD) curves that were fit to retrieve diffusion constants The mean diffusion constant of QD-EGF-EGFR was $0.021 \pm 0.022$ $\mu^{2} / \mathrm{sec}$ on the cell body and $0.015 \pm 0.013 \mu \mathrm{m}^{2} / \mathrm{sec}$ on filopodia; ie. there were no significant differences in the motion at short time scales for the two cases. The MSD plots indicated that in the presence of kinase inhibitor, the receptors undergo corralled diffusion, and not transport. A compete discussion of the data is presented elsewhere (K.A. Lidke et al., in preparation).

Although single particle tracking at these speeds could not be performed on a confocal raster scanning microscope, wide-field microscope tracking had the disadvantage of an imaging field limited to a focal plane with relatively low curvature in an area at the upper surface of the cell. Both of these limitations were overcome by utilizing the PAM for 
such measurements, permitting access to the whole cell via optical sectioning. A similar tracking algorithm was implemented for conjugate, non-conjugate and in-focus data acquired both for single planes and for complete $z$ stacks with $16 \mathrm{~ms}$ acquisition times and $20 \mathrm{~Hz}$ frequency.

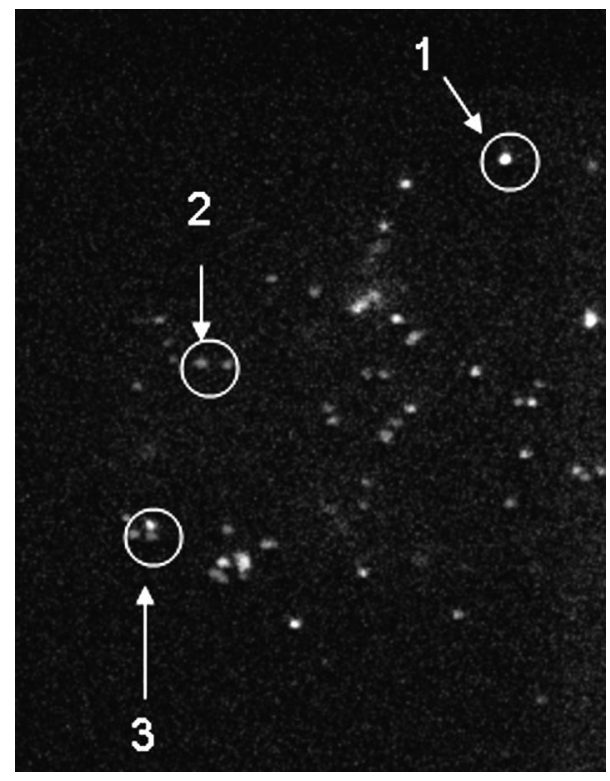

Figure 4. Movement of single QDEGF-EGFRs on an A431 cell taken with the programmable array microscope (PAM). Frames 1 (green, 0 $\mathrm{sec}$ ), 80 (red, $13.3 \mathrm{sec}$ ), and 160 (blue, $26.6 \mathrm{sec})$ of a 300 frame movie $(50 \mathrm{sec}$ total) shown in a grey-level rendition of the 3 color overlay. Diffusion and blinking of single QD-EGF-EGFR are visualized. Arrow 1 indicates a QDEGF-EGFR that did not move; arrow 2, a QD-EGF-EGFR that has blinked "off" in frame 160; and arrow 3 a mobile QDEGF-EGFR. Data were recorded with an exposure time of $33 \mathrm{~ms}$ at a rate of $\sim 6$ Hz. (taken from Hagen, et al. [15])

\subsection{FLIM imaging with the PAM}

The fluorescence emission of many fluorophores is exquisitely sensitive to their molecular micro-environment. This dependence allows one to probe the immediate surroundings at molecular distances by selective spectroscopic techniques (Förster resonance energy transfer, FRET; fluorescence lifetime imaging microscopy, FLIM) operating in the $\mathrm{nm}$ range, ie. beyond the diffraction-limited optical resolution of the microscope. The rate by which a molecule exits the excited state (monitored by the fluorescence decay) is determined by various parallel kinetic pathways. Thus, measuring the lifetime of a fluorophore reveals information about location (hydrophobicity), molecular interactions (FRET), pH, accessibility to oxygen, or solvent, and many other phenomena. A truly versatile and modern microscope should have the capability of generating images based on the distribution of intensity, spectra, polarization and lifetime. We feature here the integrated use of a commercial (LIFA, Lambert Instruments) FLIM system with the PAM.

Wide-field microscopes have been used extensively to determine fluorescence lifetimes but only recently has this measurement been combined with confocal or other sectioning techniques. There are several ways in which optically sectioned lifetime imaging can be achieved. The commercial time-domain systems rely on point scanning systems and pulsed light sources with detection either by time-correlated single photon detection (TCSP) or "boxcar" techniques [28]. The TCSP procedure is inherently accurate and sensitive but slow. Optically sectioned FLIM images are required in many research and biotechnological applications for which rapid acquisition is an important requirement. Frequency domain measurements are inherently fast [29] and we have used this in a PAM so as to achieve sectioned lifetime imaging [18]. Figure 5 features FLIM implemented in the new LCoS PAM by attaching a commercial phase modulated detector system (LIFA, Lambert Instruments).

The PAM can accommodate high-frequency modulated LEDs and lasers for FLIM. A requirement is that the intensity modulated light source for the FLIM measurements be synchronized with the patterns of illumination of the SLM. The optically sectioned measurements provide improved contrast in the lifetime images of heterogeneous systems of fluorophores, an important feature that facilitates subsequent global analysis procedures and obviates the need for recording multiple image planes. 


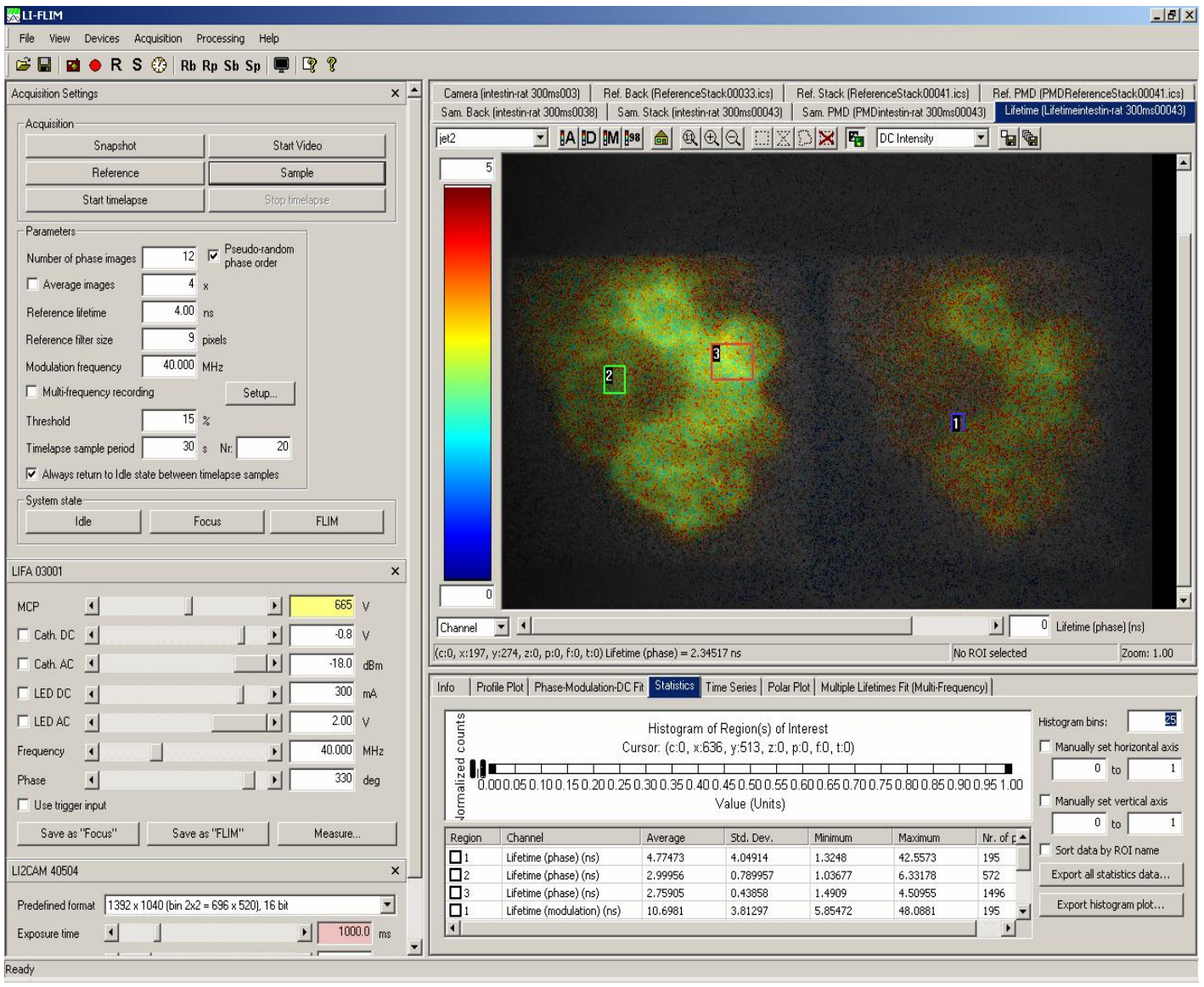

Figure 5. FLIM image of EG33-A431 cells expressing EGFR-eGFP taken with the PAM by coupling a LIFA FLIM module (Lambert Instruments). Screen shot of the data acquisition parameters and lifetime - intensity overlay image showing non-conjugate (left) and conjugate (right) images. The light source was a $470 \mathrm{~nm}, 3 \mathrm{~W}$ Lumiled (Luxeon) LED modulated at 40 MHz. 12 phase steps were recorded in psudeo-random order with an exposure of $1 \mathrm{~s}$ per step. Regions of interest were drawn, and a lifetime of $2.8 \pm 0.4$ ns was measured for eGFP (region 3). These data are preliminary and do not represent the anticipated spatial resolution with the PAM.

\subsection{Photoconversion of biomolecules with the PAM}

Photoactivation and photoconversion are important tools in cell biology for studying the kinetics of macromolecular activity or interactions. In addition, the ability to uncage active compounds at specific time and place has proven to be of immense importance for understanding fast, triggered cell responses. We showed the versatility of photoactivation and photoconversion using a PAM in a previous publication [14]. Here we demonstrate that we can initiate a complex pattern of photoconversion using the PAM that cannot be achieved with other types of microscopes. Recently Ando et al. introduced a photochromic visible fluorescent protein named DRONPA [30] which can be up-converted in a few picoseconds to a fluorescent molecule by excitation with light at $400 \mathrm{~nm}$. The photoisomerized molecule can be monitored by low-light excitation at $490 \mathrm{~nm}$, emitting fluorescence at $515 \mathrm{~nm}$, and back-converted by exposure to intense illumination at $490 \mathrm{~nm}$. The protein cycles with high quantum efficiency for many thousands of conversions. 
Figure 6 shows the conjugate and non-conjugate images generated by a complex excitation pattern (the logo of the Max Planck Society, a Minerva head) generated with the PAM using a $405 \mathrm{~nm}$ diode laser and monitoring with the 488 $\mathrm{nm}$ line of an argon ion laser. A major virtue of the photochromic process is its reversibility, allowing arbitrary patterns to be generated, erased, or reformed at will.

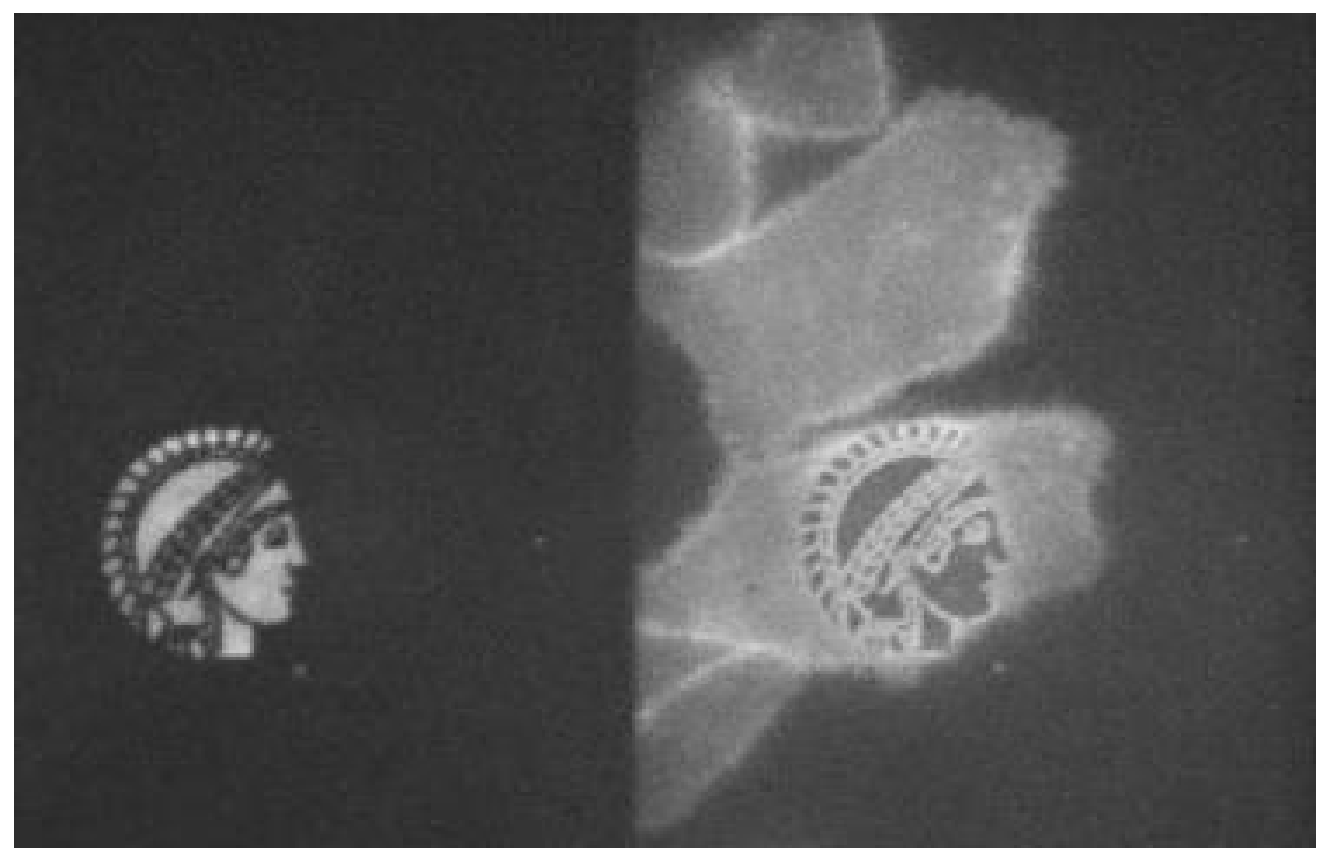

Figure 6. DRONPA forward conversion. Conjugate (left) and non-conjugate (right) images of an A431 cell expressing the erbB3-DRONPA membrane receptor. The image was generated by exciting a pattern of the Minerva with a 405 diode laser (Coherent Cube) and monitoring of the fluorescence with a full field 488 laser excitation. Images are contrast stretched.

Applications are not limited to studies of such proteins but are wide-ranging, including photolithography, diffusion studies, photomechanical action (work) and induction of altered charge distribution [31,32].

\section{Concluding Remarks}

The field of cellular imaging has benefited enormously from parallel technological developments leading to dramatic increases in sensitivity, spatial and temporal resolution, and selectivity. Luminescent quantum dots, as well as silica-based nanoparticles and nanodot clusters of noble metals not featured in this article (see reference [33]), provide single molecule sensitivity in imaging systems designed for studies of living cells [15]. We favor wide-field microscope systems for these applications because of their much higher acquisition speed, particularly in combination with electron multiplying CCD cameras that afford the ultimate detection performance at light levels of $<100$ photons/pixel. The programmable array microscope featured here offers optical sectioning in combination with high resolution in time (fluorescence lifetimes), anisotropy (FRET, diffusion), space (hyperspectral imaging, diffusion), and "chemistry" (photoreactions). The PAM should find ready use in live cell and tissue biology, real-time medical diagnostic procedures, and analytical/biotechnological applications such as in-situ hybridization and readout of biochemical arrays on chips. 


\section{Acknowledgements}

The authors acknowledge support for development of the PAM through the Max Planck Society and EU FP6 Project 037465 FLOUROMAG. BR was supported by a TALENT fellowship from the Netherlands OSR.

\section{REFERENCES}

[1] Y. Yarden and M. X. Sliwkowski, "Untangling the ErbB signalling network," Nat Rev Mol Cell Biol, vol. 2, pp. 127-137, 2001.

[2] D. J. Arndt-Jovin, D. S. Lidke, K. A. Lidke, B. Rieger, and T. M. Jovin, "Quantum dots shed light on processes in living cells," doi, 10.1117/2.1200605.0228 SPIE Newsroom, pp. 1-3, 2006.

[3] D. S. Lidke, K. A. Lidke, B. Rieger, T. M. Jovin, and D. J. Arndt-Jovin, "Reaching out for signals: filopodia sense EGF and respond by directed retrograde transport of activated receptors," J Cell Biol, vol. 170, pp. 619$626,2005$.

[4] D. S. Lidke, P. Nagy, R. Heintzmann, D. J. Arndt-Jovin, J. N. Post, H. Grecco, and T. M. Jovin, "Quantum dot ligands provide new insights into erbB/HER receptor-mediated signal transduction," Nat Biotechnol, vol. 22, pp. 198-203, 2004.

[5] Q. S. Hanley, P. J. Verveer, and T. M. Jovin, "Optical sectioning fluorescence spectroscopy in a programmable array microscope," Appl Spectrosc, vol. 52, pp. 783-789, 1998.

[6] P. J. Verveer, Q. S. Hanley, P. W. Verbeek, L. J. van Vliet, and T. M. Jovin, "Theory of confocal fluorescence imaging in the programmable array microscope (PAM)," J Microsc, vol. 189, pp. 192-198, 1998.

[7] T. M. Jovin, Q. S. Hanley, and P. J. Verveer, "Programmable Spatially Light Modulated Microscope and Microscopy," in US Patent 6,399,935. United States, 2002.

[8] T. M. Jovin, Q. S. Hanley, and P. J. Verveer, "Confocal Spectroscopy. System and Method," in US Patent 6,128,077. United States, 2000.

[9] T. M. Jovin, Q. S. Hanley, and P. J. Verveer, "Programmable Array Microscope.," in EP 0911667 Al. European Union, 2003.

[10] T. M. Jovin, Q. S. Hanley, and P. J. Verveer, "Confocal Spectroscopy. System and Method," in EP 0916981 A1. European Union, 2004.

[11] Q. S. Hanley and T. M. Jovin, "Highly multiplexed optically sectioned spectroscopic imaging in a Programmable Array Microscope," Appl Spectrosc, vol. 55, pp. 1115-1123, 2001.

[12] Q. S. Hanley, P. J. Verveer, D. J. Arndt-Jovin, and T. M. Jovin, "Three-dimensional spectral imaging by Hadamard transform spectroscopy in a programmable array microscope," J Microsc, vol. 197, pp. 5-14, 2000.

[13] R. Heintzmann, Q. S. Hanley, D. J. Arndt-Jovin, and T. M. Jovin, "A dual path Programmable Array Microscope (PAM): Simultaneous acquisition of conjugate and non-conjugate images," J Microsc, vol. 204, pp. 119-135, 2001.

[14] M. Fulwyler, Q. S. Hanley, C. Schnetter, I. T. Young, E. A. Jares-Erijman, D. J. Arndt-Jovin, and T. M. Jovin, "Selective photoreactions in a Programmable Array Microscope (PAM): Photoinitiated polymerization, photodecaging and photochromic conversion," Cytometry, vol. 67A, pp. 68-75, 2005.

[15] G. M. Hagen, K. A. Lidke, B. Rieger, D. S. Lidke, W. Caarls, D. J. Arndt-Jovin, and T. M. Jovin, "Dynamics of membrane receptors: single molecule tracking of quantum dot liganded epidermal growth factor," in Single Molecule Dynamics, Ishii and Yanagida, Eds.: Wiley, 2007.

[16] Q. S. Hanley, P. J. Verveer, and T. M. Jovin, "Spectral imaging in a programmable array microscope by hadamard transform fluorescence spectroscopy," Appl Spectrosc, vol. 53, pp. 1-10, 1999.

[17] Q. S. Hanley, D. J. Arndt-Jovin, and T. M. Jovin, "Spectrally resolved fluorescence lifetime imaging microscopy," Appl Spectrosc, vol. 56, pp. 155-166, 2002.

[18] Q. S. Hanley, K. A. Lidke, R. Heintzmann, D. J. Arndt-Jovin, and T. M. Jovin, "Fluorescence lifetime imaging in an optically sectioned Programmable Array Microscope (PAM)," Cytometry, vol. 67A, pp. 112-118, 2005.

[19] Q. S. Hanley, P. J. Verveer, M. J. Gemkow, D. Arndt-Jovin, and T. M. Jovin, "An optical sectioning programmable array microscope implemented with a digital micromirror device," J Microsc, vol. 196, pp. 317331, 1999. 
[20] S. Cha, L. PC, 1. Zhu, P.-C. Sun, and Y. Fainman, "Nontranslational three-dimensional profilometry by chromatic confocal microscopy with dynamically configurable micromirror scanning," Appl. Opt, vol. 39, pp. 2605-2613, 2000.

[21] T. Fukano and A. Miyawaki, "Whole-field fluorescence microscope with digital micromirror device: imaging of biological samples," Appl. Opt, vol. 42, pp. 4119-4124, 2003.

[22] M. Liang, R. Stehr, and Krause, AW, "Confocal pattern period in multiple-aperture confocal imaging systems with coherent illumination," Opt. Let, vol. 22, pp. 751-53, 1997.

[23] P. Smith, C. Taylor, A. Shaw, and E. McCabe, "Programmable array microscopy with a ferroelectric liquidcrystal spatial light modulator.," Appl. Opt, vol. 39, pp. 2664-2669, 2000.

[24] M. Neil, R. Juskaitis, and T. Wilson, "Method of obtaining optical sectioning by using structured light in a conventional microscope," Opt. Lett, vol. 22, pp. 1905-1907, 1007.

[25] R. Heintzman and P. Benedetti, "High-resolution image reconstruction in fluorescence microscopy with patterned excitation," Appl. Opt, vol. 45, pp. 5037-5045, 2006.

[26] I. Buck, T, D. Hom, J. Sugerman, K. Fatahalian, M. Houston, and P. Hanrahan, "Brook for GPUs: Stream computing on graphics hardware," ACM Trans. Graph, vol. 23, pp. 777-786, 2004.

[27] K. A. Lidke, B. Rieger, T. M. Jovin, and R. Heintzmann, "Superresolution by localization of quantum dots using blinking statistics," Opt Express, vol. 13, pp. 7052-7062, 2005.

[28] K. Suhling, P. French, and D. Phillips, "Lifetime Imaging microscopy," Photochem Photobiol Sci, vol. 4, pp. $13-22,2005$.

[29] R. Clegg, O. Holub, and C. Gohlke, "Fluorescence LIfetime-Resolved Imaging: Measuring lifteimes in an image," Meth. Enzymol., vol. 360, pp. 509-542, 2003.

[30] Ando R, Mizuno H, and A. Miyawaki, "Regulated fast nucleocytoplasmic shuttling observed by reversible protein highlighting.," Science, vol. 306, pp. 1370-1373, 2004.

[31] T. Sakata, Y. Yan, and G. Marriott, "Optical switching of dipolar interactions on proteins," Proc. Natl. Acad. Sci. USA, vol. 102, pp. 4759-4764, 2005.

[32] T. Sakata, Y. Yan, and G. Marriott, "Family of site-selective molecular optical switches," J Org Chem, vol. 70, pp. 2009-2013, 2005.

[33] D. J. Arndt-Jovin, M. A. Lopez-Quintela, D. S. Lidke, M. J. Rodriguez, F. M. Santos, K. A. Lidke, G. M. Hagen, and T. M. Jovin, "In vivo cell imaging with semiconductor quantum dots and noble-metal nanodots," Proceedings SPIE, vol. 6096, pp. 60960P1- 60690P10, 2006. 